BRUNA BARBOSA HACKBARTH

JeSSICA AuGustini FerReIRA ${ }^{2}$

Heidi Pfutzenreuter Carstens ${ }^{3}$

Augusto RADUnZ AMARAL ${ }^{4}$

MARIANA RIBEIRO SIIVA ${ }^{4}$

JeAN CARL Sillva 5,6

Paulo Henrique Condeixa de França ${ }^{6}$

\title{
Suscetibilidade à prematuridade: investigação de fatores comportamentais, genéticos, médicos e sociodemográficos
}

\author{
Preterm birth susceptibility: investigation of behavioral, \\ genetic, medical and sociodemographic factors
}

Artigo Original

Palavras-chave

Prematuro

Fatores de risco

Polimorfismo de nucleotídeo único Receptores de progesterona

Receptores do FSH

Keywords

Infant, premature

Risk factors

Polymorfism, single nucleotide

Receptors, progesterone Receptor, FSH

\section{Resumo}

OBJETIVO: Investigar a associação entre fatores de risco genéticos, comportamentais, biológicos e médicos e a ocorrência da prematuridade. MÉTODOS: Realizou-se estudo retrospectivo do tipo caso-controle. A técnica de reação em cadeia da polimerase em tempo real foi utilizada para analisar a influência dos polimorfismos rs 12473815 do gene codificante para o receptor do hormônio folículo estimulante (FSHR) e rs 1942836 do gene codificante para o receptor da progesterona (PGR). A avaliação dos outros fatores de risco se deu por meio da aplicação de questionários validados ou especificamente desenvolvidos e análise de dados em prontuário eletrônico. Foram incluídas 157 gestantes (45 casos com gestação <37 semanas e 112 controles com gestação >37 e $\leq 42$ semanas). RESULTADOS: Os genótipos CT do polimorfismo rs 12473815 e TC e CC do polimorfismo rs 1942836 mostraram-se associados a uma maior chance de desenvolver parto prematuro. Observou-se associação entre o nascimento prematuro e a ingestão alcoólica quando o consumo ocorreu em duas ou mais ocasiões mensais. $\bigcirc$ baixo índice de massa corporal prégestacional se mostrou preditor do nascimento prematuro espontâneo, enquanto o elevado índice de massa corporal reduziu a sua probabilidade. CONCLUSÕES: Os resultados encontrados sugerem que a ingestão alcoólica excessiva, - baixo índice de massa corporal pré-gestacional e os alelos de risco dos polimorfismos rs 12473815 e rs 1942836 dos genes FSHR e $P G R$, respectivamente, influenciam a ocorrência de nascimento prematuro.

\section{Abstract}

PURPOSE: To investigate the association between genetic, behavioral, biological and medical risk factors and the occurrence of preterm birth. METHODS: A retrospective case-control study was conducted. The real-time polymerase chain reaction was used to analyze the influence of the rs 12473815 polymorphism of the follicle stimulating hormone receptor gene (FSHR) and the rs 1942836 polymorphism of the progesterone receptor gene (PGR). Other proposed risk factors were assessed using validated or specifically developed questionnaires and analysis of electronically recorded medical data. A total of 157 patients were included (45 cases who went into labor before 37 weeks of pregnancy and 112 controls who went into labor after 37 and before 42 weeks of pregnancy). RESULTS: The genotypes CT of rs 12473815 and CT and CC of rs 1942836 were associated with a higher chance of premature delivery. There was an association between preterm birth and alcohol intake when consumption occurred 2 or more times per month. Low pre-pregnancy body mass index was a predictor of spontaneous preterm birth, while high body mass index reduced this likelihood. CONCLUSIONS: The results suggest that excessive alcohol intake, a low level of pre-pregnancy body mass and the risk alleles of rs 12473815 and rs 1942836 polymorphisms of the FSHR and PGR genes, respectively, influence the occurrence of preterm birth.
Correspondência

Bruna Barbosa Hackbarth Rua Paulo Malschitzki, 10 - Bom Retiro CEP: $89219-710$ Joinville (SC), Brasil

Recebido $25 / 03 / 2015$

Aceito com modificacões

$24 / 06 / 2015$
Programa de Pós-graduação em Saúde e Meio Ambiente da Universidade da Região de Joinville - UNIVILLE - Joinville (SC), Brasil. 'Programa de Pós-graduação em Saúde e Meio Ambiente, Universidade da Região de Joinville - UNIVILLE - Joinville (SC), Brasil. ${ }^{2}$ Curso de Graduação, Departamento de Farmácia, Universidade da Região de Joinville - UNIVILLE - Joinville (SC), Brasil. ${ }^{3}$ Departamento de Farmácia, Universidade da Região de Joinville - UNIVILLE - Joinville (SC), Brasil. ${ }^{4}$ Curso de Graduação em Medicina, Departamento de Medicina, Universidade da Região de Joinville - UNIVILLE - Joinville (SC), Brasil. ${ }^{5}$ Serviço de Gestação de Alto Risco, Maternidade Darcy Vargas - Joinville (SC), Brasil.

${ }^{\circ}$ Departamento de Medicina, Universidade da Região de Joinville - UNIVILLE - Joinville (SC), Brasil.

Conflito de interesses: não há 
Introdução

A Organização Mundial de Saúde (OMS) define como prematuro todo recém-nascido $(\mathrm{RN})$ vivo oriundo de gestação anterior à $37^{\mathrm{a}}$ semana ${ }^{1}$. De acordo com a semana gestacional em que ocorre o nascimento, os casos de prematuridade podem ser classificados em limítrofes $\left(35^{a}-<37^{a}\right.$ semana), intermediários $\left(32^{a}-<35^{a}\right.$ semana), muito prematuros $\left(28^{\mathrm{a}}-<32^{\mathrm{a}}\right.$ semana) e extremos $\left(<28^{a} \text { semana }\right)^{2}$.

$\mathrm{Na}$ maior parte dos países com estudos sobre a matéria, vem se percebendo um aumento nas taxas de prematuridade. Em 2010, 15 milhões de bebês nasceram prematuramente, sugerindo uma prevalência mundial de $11,1 \%{ }^{3}$. Em 2005, essa prevalência era de $9,6 \%{ }^{1}$. O Brasil, $10^{\circ}$ país com maior número de casos estimados de prematuridade, apresenta cenário semelhante ${ }^{3}$. Estudos epidemiológicos mostram que a prevalência do nascimento prematuro vem aumentando desde 1990, tendo alcançado taxas de até $15 \%$ em algumas cidades do Sul e Sudeste do país em $2004^{3,4}$. Um estudo de coorte brasileiro identificou a prematuridade como principal causa de morte neonatal ${ }^{5}$.

$\mathrm{Na}$ tentativa de reverter esse quadro, pesquisadores voltaram-se à identificação das causas do nascimento prematuro. Até o momento, são estudados os aspectos comportamentais e sociodemográficos, as condições médicas prévias ou complicações da gravidez e, mais recentemente, os fatores genéticos ${ }^{6}$.

A prematuridade como resultado do processo evolutivo constitui um novo tipo de abordagem. Ao se comparar humanos com primatas semelhantes, identifica-se que, ao longo do tempo, a nossa espécie apresentou um aumento substancial do crânio e um estreitamento da bacia pélvica, dificultando o trabalho de parto ${ }^{7}$. Tais constatações têm impulsionado a investigação de possíveis associações entre variantes de genes acelerados e a ocorrência de prematuridade. Plunkett et al. ${ }^{7}$ sugeriram que os genes FSHR e PGR, codificantes para os receptores do hormônio folículo estimulante e da progesterona, respectivamente, evoluíram de maneira acelerada na espécie humana, impactando o encurtamento da gestação e a facilitação do nascimento ${ }^{7}$. Até o momento, já foi identificada associação significativa entre a prematuridade e os polimorfismos de nucleotídeo único (SNPs - Single Nucleotide Polymorphism) rs12473815 do gene FSHR e rs1942836 do gene PGR em algumas populações $^{7-9}$.

O objetivo do estudo foi investigar a associação de certas características maternas, como consumo de álcool, uso de tabaco, escolaridade, condição socioeconômica, índice de massa corporal (IMC) pré-gestacional, diabetes gestacional (DG), ocorrência de infecções durante a gestação e a presença das variantes de risco relativas aos SNPs rs 12473815 e rs 1942836 , com o nascimento prematuro.
Métodos

O estudo retrospectivo do tipo caso-controle foi realizado na Maternidade Darcy Vargas (MDV), localizada em Joinville, Santa Catarina. A instituição é referência na assistência pré-natal e puerperal de toda a região norte do estado oriundos do Sistema Único de Saúde (SUS).

A inclusão de pacientes e a coleta de dados ocorreram entre os meses de outubro de 2013 e outubro de 2014 . Durante o período estipulado, 157 puérperas foram inclusas no estudo, sendo $45(28,6 \%)$ com trabalho de parto prematuro (grupo "Caso") e $112(71,4 \%)$ com trabalho de parto a termo (grupo "Controle"). Ambos os grupos apresentavam média de idade equivalente, sendo $23,3 \pm 4,0$ anos para o grupo Caso e 23,3 $\pm 3,9$ anos para o Controle.

Foram inclusas, consecutivamente, puérperas primíparas entre 18 e 35 anos, atendidas na MDV com trabalho de parto espontâneo, resultando em $\mathrm{RN}$ único, vivo e sem malformações evidentes. Por meio da confirmação da idade gestacional por ultrassonografia, consideraram-se casos prematuros os partos ocorridos anteriormente à $37^{\mathrm{a}}$ semana. Para o Grupo Controle, incluíram-se partos ocorridos entre a $37^{\mathrm{a}}$ e a $42^{\mathrm{a}}$ semana de gestação.

Após anuência da puérpera, realizou-se entrevista individualizada durante o período de internação pós-parto, empregando-se questionário de pesquisa composto por instrumentos já validados e por perguntas especialmente elaboradas para o estudo, além de consulta ao prontuário médico respectivo. Todas as informações coletadas foram digitadas em banco de dados utilizando-se o programa Epidata 3.1 ${ }^{\circledR}$.

Para avaliar o consumo de álcool, utilizou-se o instrumento AUDIT-C (Alcobol Use Disorders Identification Test $C$ ). As qualidades psicométricas desse questionário, já testadas para gestantes, identificam o consumo elevado de álcool com uma sensibilidade de 94,6\% quando seus resultados são maiores ou iguais a $3^{10}$.

A obtenção dos dados socioeconômicos foi definida pelo Critério de Classificação Econômica Brasil (CCEB) da Associação Brasileira das Empresas de Pesquisa ${ }^{11}$. Por meio do CCEB, atribui-se à participante uma pontuação que a classifica em estratos econômicos ${ }^{11}$. Para a definição da escolaridade da puérpera, empregaram-se as mesmas categorias contidas no CCEB.

Em relação à ocorrência de infecções durante a gestação, elaborou-se questionário contendo perguntas que abordavam os aspectos mais pertinentes, de acordo com revisão bibliográfica. Dentre eles, destacam-se o sítio da infecção apresentada, em qual trimestre gestacional se deu a ocorrência e se houve a necessidade de antibioticoterapia.

Quanto ao uso do tabaco, por não se dispor de um questionário padronizado e específico para essa população, foram investigados aspectos do tabagismo materno descritos em estudo realizado no extremo sul brasileiro ${ }^{12}$. Indagou-se 
se a participante fumou nos seis meses que antecederam a gravidez; se fumou durante a gravidez e em qual trimestre; e se seu companheiro ou outro familiar costumava fumar na mesma peça do domicílio em que ela se encontrava durante a gestação. Em caso de respostas afirmativas, as participantes eram consideradas tabagistas ativas ou passivas.

Em relação ao IMC, optou-se pela coleta de informações autorreferidas, a fim de se evitar a realização de manobras dolorosas para a medição da altura da paciente no pós-parto. Para a captação dos dados relacionados ao DG foram utilizadas as informações contidas nos prontuários médicos.

\section{Análise genética}

Para as análises genéticas foram utilizadas alíquotas residuais de sangue total sem aditivo, coletado para realização do teste VDRL (Veneral Disease Research Laboratory) na admissão hospitalar. A extração do DNA seguiu as recomendações do kit "Biopur Kit de Extração Mini Spin Plus" (Biopur, Reinach, Suíça). Em seguida, realizou-se a discriminação alélica dos polimorfismos rs12473815 e rs1942836 através do sistema Taqman $^{\circledast}$ para PCR em Tempo Real (qPCR) (Life Technologies, Carlsbad, EUA). Na reação empregaram-se sondas com os fluoróforos reporter $\mathrm{VIC}^{\circledR} \mathrm{e} \mathrm{FAM}^{\circledR}$. Para a preparação de ambas as reações, estimou-se volume total de $25 \mu \mathrm{L}$, conforme a seguinte proporção: $100 \mathrm{ng}$ de amostra de DNA, 12,5 $\mu \mathrm{L}$ de Taqman ${ }^{\circledR}$ Genotyping Master Mix (2X), 1,25 $\mu \mathrm{L}$ de TaqMan ${ }^{\circledR}$ SNP Genotyping Assay 4351379 (20X) e q.s.p. $\mathrm{H}_{2} 0$ DEPC (Diethylpyrocarbonate). As reações foram distribuídas em placa para leitura óptica (MicroAmp ${ }^{\circledR}$ Optical 96-well reaction plate) e levadas ao termociclador modelo CFX-96 Touch (Bio-Rad, Hercules, EUA). Utilizou-se a seguinte condição de termociclagem: 10 minutos a $95^{\circ} \mathrm{C}, 39$ ciclos alternados de 15 segundos a $95^{\circ} \mathrm{C}$ e um minuto a $60^{\circ} \mathrm{C}$. O resultado das análises foi avaliado mediante gráficos de discriminação alélica, construídos pelo próprio software do equipamento.

\section{Análise estatística}

Para a análise descritiva, realizaram-se as medidas de média, desvio padrão e frequências absoluta e relativa. Inicialmente, foi avaliada a normalidade da distribuição dos dados utilizando-se o teste de Komolgorov-Smirnov. Aplicou-se o teste $t$ para a análise da hipótese de igualdade entre as médias geradas a partir da variável idade materna. As frequências alélicas e genotípicas foram calculadas a partir de contagem direta e comparadas entre os grupos Caso e Controle. A partir daí, testou-se a aderência dos dados ao Equilíbrio de Hardy-Weinberg. Para a comparação das proporções entre os dois grupos, realizou-se teste $\chi^{2}$ ou teste exato de Fisher. Foram construídos modelos de regressão logística para avaliar a associação dos fatores de risco estudados com a prematuridade e ajustar as variáveis de confundimento. O intervalo de confiança (IC) estabelecido foi de $95 \%$ e significância quando $\mathrm{p}<0,05$.
A análise estatística foi realizada empregando-se o Software SPSS (Statistical Package for the Social Sciences) versão 21.0.

O estudo foi aprovado pelo Comitê de Ética em Pesquisa da Universidade da Região de Joinville (parecer 392.814) e respeitou as normas contidas na Resolução CNS 466/2012 e complementares. A participação das puérperas se deu mediante assinatura de termo de consentimento livre e esclarecido específico.

\section{Resultados}

Dentre os nascimentos do grupo Caso, observou-se uma maior prevalência de prematuridade limítrofe (35 casos; $77,8 \%)$, seguida de 4 casos de prematuros moderados $(8,9 \%)$, 3 de muito prematuros $(6,7 \%)$ e 1 de prematuridade extrema $(2,2 \%)$. Em dois eventos de nascimento prematuro (4,4\%), a idade gestacional não foi informada no prontuário eletrônico. No entanto, diante da importância das informações fornecidas pela aplicação do questionário e do teste genético, optou-se por mantê-las no estudo.

Em relação à ocorrência de infecção microbiana, não se observou diferença significativa entre puérperas com parto prematuro $(n=21 ; 46,7 \%)$ e a termo $(n=49$; $43,7 \%)(\mathrm{p}=0,9)$. Dentre as participantes da pesquisa, $5(3,3 \%)$ apresentavam DG. Destas, 4 pertenciam ao grupo Controle e 1 ao grupo Caso. A escolaridade, os estratos de condição socioeconômica e o comportamento tabagista também não tiveram influência sobre o desfecho estudado. Os resultados referentes à ingestão alcoólica e ao IMC pré-gestacional, entretanto, mostraram associação positiva com o nascimento prematuro (Tabela 1).

O consumo de álcool se tornou relevante somente com a ingestão superior a duas doses mensais. O teste AUDIT-C foi capaz de identificar a ingestão alcoólica excessiva como possível fator de risco para o nascimento prematuro. No grupo Caso, a ocorrência de puérperas com pontuação $\geq 3(17,8 \%)$ foi superior à encontrada no grupo Controle $(6,3 \%)(\mathrm{p}=0,03)$. Da mesma forma, o baixo IMC pré-gestacional $\left(<18,5 \mathrm{~kg} / \mathrm{m}^{2}\right)$ aumentou em

Tabela 1. Influência do tabagismo, da ingestão alcoólica e do índice de massa corporal pré-gestacional sobre a frequência de nascimentos prematuros

\begin{tabular}{|c|c|c|c|c|c|c|}
\hline & \multicolumn{2}{|c|}{ Controle } & \multicolumn{2}{|c|}{ Caso } & \multirow{2}{*}{ OR } & \multirow{2}{*}{ IC95\% } \\
\hline & $n$ & $\%$ & $n$ & $\%$ & & \\
\hline Tabagismo ativo & 8 & 7,1 & 6 & 13,3 & 2,7 & $0,6-9,8$ \\
\hline Tabagismo passive & 64 & 57,1 & 26 & 57,7 & 1,0 & $0,5-1,9$ \\
\hline $\begin{array}{l}\text { Consumo alcoólico mensal } \\
\geq 2 \text { vezes }\end{array}$ & 40 & 35,7 & 21 & 46,6 & 1,7 & $1,1-4,6^{*}$ \\
\hline $\begin{array}{l}\text { IMC baixo peso } \\
\left(<18,5 \mathrm{~kg} / \mathrm{m}^{2}\right)\end{array}$ & 8 & 7,1 & 9 & 20 & 3,8 & $1,3-11,6^{\star}$ \\
\hline $\begin{array}{l}\text { IMC excesso de peso } \\
\left(\geq 25,0 \mathrm{~kg} / \mathrm{m}^{2}\right)\end{array}$ & 34 & 30,3 & 11 & 24,4 & 0,6 & $0,3-0,9 *$ \\
\hline
\end{tabular}

OR: Odds ratio; IC: Intervalo de confiança; IMC: Índice de massa corporal; * Significância estatística. 
3,8 vezes a chance de se desenvolver um parto prematuro (IC95\% 1,3-11,6). Em oposição, o excesso de peso pré-gestacional $\left(\mathrm{IMC}>25,0 \mathrm{~kg} / \mathrm{m}^{2}\right)$ apresentou efeito protetor sobre a prematuridade (IC95\% 0,3-0,9).

Para a interpretação dos dados genéticos, três amostras foram excluídas da análise por apresentarem falha não identificável durante o processo de extração de DNA ou resultados inconclusivos após qPCR. Dessa maneira, 154 amostras foram consideradas em relação aos polimorfismos genéticos rs12473815 (45 controles e 109 casos) e rs1942836 (44 casos e 110 controles). Ambos os grupos se encontravam em equilíbrio de Hardy-Weinberg. Quando comparadas as frequências genotípicas e alélicas de ambos os polimorfismos entre os grupos, não se evidenciaram diferenças significativas em relação a sua distribuição (Tabela 2).

IMC pré-gestacional, etilismo e tabagismo foram considerados como potenciais fatores confundidores e incluídos na análise multivariada. Nessa etapa, identificou-se que os genótipos TC e CC do SNP rs1942836 do gene PGR aumentaram em 1,4 (IC95\% 1,1-1,9) e em 4,4 (IC95\% 1,3-17,8) vezes, respectivamente, as chances de se desenvolver parto prematuro. Em relação ao gene FSHR, não foram detectadas gestantes homozigotas para a variável de risco do SNP rs12473815. No entanto, observou-se que gestantes com o genótipo CT apresentaram chances 1,6 (IC95\% 1,2-3,3) vezes maiores de apresentarem prematuridade. Quando analisados os efeitos combinados de ambos os polimorfismos (rs12473815 e rs1942836) sobre o desfecho, foi observada maior ocorrência de prematuridade nas gestantes portadoras dos genótipos CT/TC (OR 2,0; IC95\% 1,1-3,2) e CT/CC (OR 1,4; IC95\% 1,1-2,5) (Tabela 3).

Tabela 2. Frequências genotípicas e alélicas dos polimorfismos rs 12473815 do gene FSHR e rs 1942836 do gene PGR

\begin{tabular}{|c|c|c|c|c|c|}
\hline \multirow{4}{*}{$\begin{array}{c} \\
\text { FSHR } \\
\text { Genóti }\end{array}$} & \multicolumn{2}{|c|}{ Caso } & \multicolumn{2}{|c|}{ Controle } & \multirow{2}{*}{ Valor $p$} \\
\hline & \multirow[t]{2}{*}{$n$} & \multirow[t]{2}{*}{$\%$} & \multirow[t]{2}{*}{$\mathrm{n}$} & \multirow[t]{2}{*}{$\%$} & \\
\hline & & & & & \\
\hline & & & & & \\
\hline CC & 21 & 46,7 & 43 & 39,5 & $0,4^{*}$ \\
\hline СТ & 24 & 53,3 & 66 & 60,5 & \\
\hline \multicolumn{6}{|l|}{ Alelo } \\
\hline C & 66 & 73,3 & 152 & 69,7 & $0,4^{\star}$ \\
\hline$T$ & 24 & 26,7 & 66 & 30,3 & \\
\hline \multicolumn{6}{|l|}{ PGR } \\
\hline \multicolumn{6}{|c|}{ Genótipo } \\
\hline TT & 24 & 54,5 & 73 & 66,3 & $0,2^{\star \star}$ \\
\hline TC & 16 & 36,4 & 32 & 29,1 & \\
\hline $\mathrm{CC}$ & 4 & 9,1 & 5 & 4,6 & \\
\hline \multicolumn{6}{|l|}{ Alelo } \\
\hline $\mathbf{T}$ & 64 & 72,7 & 178 & $0,11^{*}$ & $0,1^{\star}$ \\
\hline C & 24 & 27,3 & 42 & 19,1 & \\
\hline
\end{tabular}

FSHR: Receptor do hormônio folículo estimulante; PGR: Receptor de progesterona;

*Teste $\chi^{2} ; * \star$ Teste exato de Fisher.
Tabela 3. Análise multivariada da ocorrência de prematuridade de acordo com a apresentaccão das variantes dos polimorfismos dos genes FSHR (rs1 2473815) e PGR (rs 1942836)

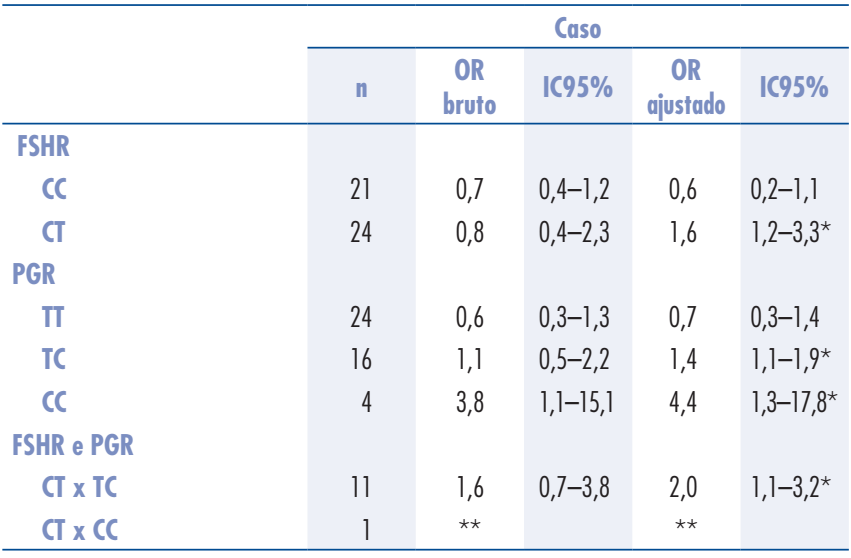

OR: Odds Ratio; IC: Intervalo de confiança; FSHR: Receptor do hormônio folículo estimulante; PGR: Receptor de progesterona. * Significância estatística. * *Análise excluída em razão do baixo número amostral.

\section{Discussão}

A predominância de prematuridade limítrofe encontrada no estudo é comparável a dados obtidos em revisão sistemática, em que se observaram prevalências de 63,2\%, $76 \%$ e $79 \%$ em três diferentes populações ${ }^{13}$.

Neste estudo, o consumo alcoólico aumentou as chances de nascimento prematuro somente quando a ingestão ocorria em duas ou mais ocasiões por mês. Até o momento, entretanto, a maior parte das publicações mostra a necessidade de doses ainda maiores e mais frequentes para predispor à prematuridade. Uma revisão sistemática com metanálise realizada em 2011 identificou que apenas o consumo a partir de três doses diárias (ou $36 \mathrm{~g} / \mathrm{dia}$ ) aumentaria as chances de um parto prematuro ${ }^{14}$.

A influência deambos os extremos do IMC pré-gestacional sobre a prematuridade já foi relatada na literatura ${ }^{15}$. Dessa forma, o efeito protetor do excesso de peso encontrado no presente estudo deve-se, possivelmente, a um viés de seleção, relacionado aos critérios de inclusão do estudo. Sabe-se que os extremos superiores do IMC pré-gestacional estão relacionados ao parto prematuro por Ruptura Prematura de Membrana ou por indicação médica, ambas as modalidades não admitidas no estudo ${ }^{16}$. Tal associação já foi verificada em coorte estadunidense ao se verificar associação entre IMC $>30 \mathrm{~kg} / \mathrm{m}^{2}$ e os nascimentos prematuros por indicação médica, com OR de 1,4 nas gestações entre a $34^{a}$ e $36^{a}$ semana e de 1,7 nas anteriores à $34^{\mathrm{a}}$ semana gestacional ${ }^{16}$.

Em relação ao uso do tabaco, mesmo não se encontrando associação significativa com a prematuridade, é possível observar que há uma elevação na taxa de gestantes tabagistas no local de estudo (13,3\% no grupo Caso) quando comparada à média nacional $(7,7 \%)^{17}$. Ainda, 
é importante salientar a taxa de gestantes consideradas fumantes passivas (57\%). Recentemente, demonstrou-se associação positiva entre a inalação passiva do fumo e o nascimento prematuro, principalmente ao se considerar os partos ocorridos com idade gestacional inferior a 32 semanas $^{18}$.

A prevalência de DG encontrada no estudo $(3,3 \%)$ mostrou comportamento inverso ao apresentado pelo tabagismo. Segundo dados do Ministério da Saúde ${ }^{19}$ publicados em 2010, a taxa de prevalência de DG em mulheres com mais de 20 anos atendidas no Sistema Único de Saúde é de 7,6\%. Os dados das pacientes atendidas na MDV não correspondem à metade do valor encontrado nacionalmente. A MDV, por contar com ambulatório especializado em diabetes e com programa específico para prevenção de prematuridade, pode ter apresentado número reduzido de casos de DG severo ou descompensado, impossibilitando a verificação de sua associação com o desfecho.

A falta de associação entre escolaridade e condição socioeconômica e a prematuridade pode se dar pela sua difícil mensuração ${ }^{20}$. Em consonância com o modelo exposto pela OMS, os fatores socioeconômicos já foram identificados como determinantes estruturais do parto prematuro ${ }^{21}$. Segundo tal concepção, no entanto, eles não influenciam diretamente a condição de saúde da gestante, sendo capazes de alterar, apenas, seus aspectos comportamentais e psicossociais, os chamados determinantes intermediários. Neste estudo, elevados níveis de consumo alcoólico e o baixo IMC pré-gestacional podem ser considerados determinantes intermediários, capazes de aumentar as chances de um parto prematuro.

Os resultados encontrados sobre o polimorfismo do gene PGR confirmam os dados de estudos anteriores. Em pesquisas realizadas nos EUA, em 2007, e na Argentina, em 2013, se identificou a associação da variante de risco do SNP rs1942836 com a prematuridade limítrofe ${ }^{8,9}$. A influência dos genótipos TC e CC encontrada no presente estudo, entretanto, não se restringiu apenas a essa classe de nascimento prematuro. A associação foi identificada ao se considerar todos os tipos prematuridade, envolvendo casos limítrofes a extremos.

A influência do SNP rs12473815 sobre a prematuridade já foi observada em população finlandesa e afro-americana. Em finlandesas, analisando um grupo de 165 casos e 163 controles, observou-se diferença entre as frequências genotípicas e alélicas dos dois grupos ${ }^{7}$. $\mathrm{Na}$ população afro-americana, com 79 casos e 171 controles, também se observaram diferenças significativas entre tais frequências ${ }^{7}$.

A frequência do alelo de risco $T$ reportada em gestantes finlandesas com parto prematuro foi de $41,7 \%$, superior a das pacientes com a mesma condição neste estudo $^{22}$. Deve-se considerar que essas populações apresentam ancestralidade e características genéticas distintas. Enquanto a primeira apresenta marcada homogeneidade, a população brasileira detém, reconhecidamente, elevada miscigenação ${ }^{23}$. Tais distinções podem ser responsáveis pela ausência de genótipos homozigotos TT na população desta pesquisa.

Ainda em relação ao SNP rs12473815 do gene FSHR, curiosamente, é possível observar que o genótipo $\mathrm{CT}$ foi mais frequente no grupo Controle do que no grupo Caso. Isso se deve, possivelmente, à característica multifatorial da prematuridade. Hábitos comportamentais e condições sociodemográficas, biológicas e médicas favoráveis permitiram que gestantes, mesmo dispondo o genótipo de risco, apresentassem trabalho de parto a termo.

Replicar estudos em diferentes centros populacionais constitui um grande desafio em razão das disparidades raciais. O tempo de duração de uma gestação e as taxas de prematuridade variam de modo expressivo entre diferentes raças ${ }^{24}$. Estudo publicado em 2003, por exemplo, demonstra chance mais elevada de ocorrência de parto prematuro em negras e asiáticas, quando comparadas às gestantes brancas europeias, sugerindo que a maturação fetal em tais casos ocorre mais precocemente ${ }^{24}$. No Brasil, país de elevada miscigenação racial, é difícil estimar até que ponto tais características naturais interferem na prematuridade.

Em relação à análise dos SNPs rs12473815 do gene FHSR e rs 1942836 do gene PGR, é importante também considerar que suas indicações como fator relacionado com a prematuridade são recentes e que, portanto, poucos estudos foram realizados até o momento.

Considerando os aspectos multifatoriais que envolvem o nascimento prematuro, é possível reconhecer algumas limitações do estudo que possam ter inviabilizado a determinação de associação positiva entre alguns fatores de risco estudados e a prematuridade. Inicialmente, a entrevista das pacientes ocorreu após o parto, permitindo, por meio de viés de memória, que algumas informações já não pudessem ser recordadas. Adicionalmente, existe a possibilidade de dados terem sido deliberadamente fornecidos de maneira errônea, configurando um possível viés devido a respostas falseadas. Admite-se, ainda, que os resultados alcançados estejam sob a influência de um número reduzido de gestantes provenientes de um único centro.

Em conclusão, a ingestão frequente de álcool, os extremos do IMC pré-gestacional e a presença do genótipo CT do SNP rs12473815 do gene FSHR e dos genótipos TC e CC do SNP rs1942836 mostraram associação com o nascimento prematuro. É importante considerar que a 
prematuridade apresenta um caráter complexo e multifatorial, envolvendo diversos aspectos sociais, comportamentais e biológicos que, em estudos posteriores, poderão apresentar correlação com a prematuridade. Ao se conhecer a influência de certas características maternas, auxilia-se no planejamento e na gestão da assistência à saúde integral da gestante e do RN.

\section{Agradecimentos}

Ao Fundo de Apoio à Pesquisa da Universidade da Região de Joinville (FAP/UNIVILLE) pelo suporte financeiro e ao Conselho Nacional de Desenvolvimento Científico e Tecnológico (CNPq) pela concessão de bolsa de estudos CAPES à pesquisadora Bruna Barbosa Hackbarth.

\section{Referências}

1. Beck S, Wojdyla D, Say L, Betran AP, Merialdi M, Requejo JH, et al. The worldwide incidence of preterm birth: a systematic review of maternal mortality and morbidity. Bull World Health Organ. 2010;88(1):31-8.

2. Blencowe $H$, Cousens $S$, Oestergaard MZ, Chou D, Moller AB, Narwal R, et al. National, regional, and worldwide estimates of preterm birth rates in the year 2010 with time trends since 1990 for selected countries: a systematic analysis and implications. Lancet. 2012;379(9832):2162-72.

3. World Health Organization [Internet]. Born too soon: the global action report on preterm birth. Geneva: WHO; 2012 [cited 2014 Oct 03]. Available from: <http://www.who.int/pmnch/media/ news/2012/201204_borntoosoon-report.pdf>

4. Silveira MF, Santos IS, Barros AJD, Matijasevich A, Barros FC, Victora CG. Aumento da prematuridade no Brasil: revisão de estudos de base populacional. Rev Saúde Pública. 2008;42(5):957-64.

5. Lansky S, Friche AAL, Silva AAM, Campos D, Bittencourt SDA, Carvalho ML, et al. Pesquisa Nascer no Brasil: perfil da mortalidade neonatal e avaliação da assistência à gestante e ao recém-nascido. Cad Saúde Pública. 2014;30(Supl 1):S192-207.

6. Behrman RE, Butler AS. Preterm birth: causes, consequences and prevention [Internet]. Washington (DC): National Academies Press; 2007 [cited 2014 Sep 10]. Available from: <http://www.ncbi. nlm.nih.gov/books/NBK1 1362/>

7. Plunkett J, Doniger S, Orabona G, Morgan T, Haataja R, Hallman $M$, et al. An evolutionary genomic approach to identify genes involved in human birth timing. PLoS Genet. $2011 ; 7(4)$ :e 1001365.

8. Ehn NL, Cooper ME, Orr K, Shi M, Johnson MK, Caprau D, et al. Evaluation of fetal and maternal genetic variation in the progesterone receptor gene for contributions to preterm birth. Pediatr Res. 2007;62(5):630-5.

9. Mann PC, Cooper ME, Ryckman KK, Comas B, Gili J, Crumley S, et al. Genetic influences on preterm birth in Argentina. J Perinatol. 2013;33(5):336-40.

10. Dawson DA, Grant BF, Stinson FS, Zhou Y. Effectiveness of the derived Alcohol Use Disorders Identification Test (AUDIT-C) in screening for alcohol use disorders and risk drinking in the US general population. Alcohol Clin Exp Res. 2005;29(5):844-54.

11. Associação Brasileira de Empresas de Pesquisa. Critério de Classificação Econômica Brasil. São Paulo: ABEP; 2008.

12. Zhang L, González-Chica DA, Cesar JA, Mendoza-Sassi RA, Beskow $B$, Larentis $N$, et al. Tabagismo materno durante a gestação e medidas antropométricas do recém-nascido: um estudo de base populacional no extremo sul do Brasil. Cad Saúde Pública. $2011 ; 27(9): 1768-76$.

13. Machado-Junior LC, Passini-Junior R, Rosa IRM. Prematuridade tardia: uma revisão sistemática. J Pediatr. 2014;90(3):221-31.

14. Patra J, Bakker R, Irving H, Jaddoe VW, Malini S, Rehm J. Doseresponse relationship between alcohol consumption before and during pregnancy and the risks of low birthweight, preterm birth and small for gestational age (SGA) - a systematic review and meta-analyses. BJOG. 2011 ; 118(12):1411-21.

15. Lynch AM, Hart JE, Agwu OC, Fisher BM, West NA, Gibbs RS. Association of extremes of prepregnancy BMI with the clinical presentations of preterm birth. Am J Obstet Gynecol. 2014;210(5):428.e1-9.

16. Parker MG, Ouyang F, Pearson C, Gilman MW, Belfort MB, Hong $X$, et al. Prepregnancy body mass index and risk of preterm birth: association heterogeneity by preterm subgroups. BMC Pregnancy Childbirth. 2014;14:153.

17. Levy D, Jiang M, Szklo A, Almeida LM, Autran M, Bloch M. Smoking and adverse maternal and child health outcomes in Brazil. Nicotine Tob Res. 2013;15(1 1):1797-804.

18. Qiu J, He X, Cui H, Zhang C, Zhang H, Dang Y, et al. Passive smoking and preterm birth in urban China. Am J Epidemiol. 2014;180(1):94-102.

19. Brasil. Ministério da Saúde. Secretaria de Atenção à Saúde. Departamento de Ações Programáticas Estratégicas. Gestação de alto risco: manual técnico. 5 a ed. Brasília (DF): Ministério da Saúde; 2010.

20. Niedhammer I, Murrin C, O'Mahony D, Daly S, Morrison JJ, Kelleher CC, et al. Explanations for social inequalities in preterm delivery in the prospective Lifeways Cohort in the Republic of Ireland. Eur J Public Health. 2011 ;22(4):533-8.

21. Dolatian M, Mirabzadeh A, Forouzan AS, Sajiadi H, Majd HA, Moafi F. Preterm delivery and psycho-social determinants of health based on World Health Organization model in Iran: a narrative review. Glob J Health Sci. 2013;5(1):52-64.

22. Chun S, Plunkett J, Teramo K, Muglia L, Fay JC. Fine-mapping association of FSHR with preterm birth in a Finnish population. PLoS One. 2013;8(10):e78032.

23. Pena SD, Bastos-Rodrigues L, Pimenta JR, Bydlowski SP. DNA tests probe the genomic ancestry of Brazilians. Braz J Med Biol Res. 2009;42(10):870-6.

24. Patel RR, Steer P, Doyle P, Little MP, Elliott P. Does gestation vary by ethnic group? A London-based study over 122.000 pregnancies with spontaneous onset of labor. Int J Epidemiol. 2003;33(1):107-13. 